This document is the accepted manuscript version of the following article:

Mochi, I., \& Ekinci, Y. (2019). Lensless EUV 1ithography and imaging.

Synchrotron Radiation News, 32(4), 22-27.

https://doi .org/10.1080/08940886.2019.1634433

\title{
Lensless EUV lithography and imaging
}

Iacopo Mochi and Yasin Ekinci.

Laboratory of Micro and Nanotechnology, Paul Scherrer Institut, Villigen, Switzerland

*Iacopo Mochi. Paul Scherrer Institut, Forschungsstrasse 111. 5232 Villigen PSI

Switzerland. Phone: +41 5631045 73. E-Mail: Iacopo.Mochi@psi.ch 


\section{Lensless EUV lithography and imaging}

Keywords: extreme ultra violet; interference lithography; coherent diffraction imaging; mask inspection; resist.

\section{Introduction}

Extreme ultraviolet (EUV) radiation is a powerful tool for material characterization and for many years it has been developed as a potential candidate to replace deep ultraviolet (DUV) in the photolithography process used for semiconductor devices manufacturing for future technology nodes. In this process, a pattern engraved on a mask is exposed with light with a wavelength of $13.5 \mathrm{~nm}$ and transferred on a silicon wafer coated with an EUV photosensitive resist material ${ }^{1}$.

Recently, EUV lithography has matured enough to enter in the high-volume manufacturing phase, but to ensure a continued progress of this technology, more research is necessary for the development of EUV resists that can support higher resolution with more sensitivity. Ideally, EUV resist development and testing could be carried out using standard EUV scanners, the devices employed in semiconductor devices production facilities to transfer the patterns from the mask to the silicon wafer. Nevertheless, these devices are expensive, and their availability is limited, therefore not all EUV resist developers can get enough access to them for their research. Another problematic aspect of EUV lithography is the necessity of frequent and reliable monitoring of the mask to verify that it remains defect free during the wafer printing process. Mask manufacturing defects or fall-on particle contamination could reduce the production yield significantly. Therefore, effective metrology techniques for mask defect inspection at $13.5 \mathrm{~nm}$ wavelength are essential. Currently, no EUV mask metrology at $13.5 \mathrm{~nm}$ is commercially available, because of the technological challenges and costs. 
EUV radiation for lithography and metrology can be generated in different ways, including plasma sources, high-harmonic generation, and synchrotron sources. The latter solution allows to cover the whole EUV spectrum from $124 \mathrm{eV}$ to $10 \mathrm{eV}$. The XIL-II beamline at the Swiss Light Source (SLS) is dedicated to EUV research for semiconductor manufacturing and is equipped with two end-stations that exploit the coherence properties of the beamline to investigate new materials and to perform advanced mask metrology without the necessity of complex and expensive EUV optical systems (Figure 1).

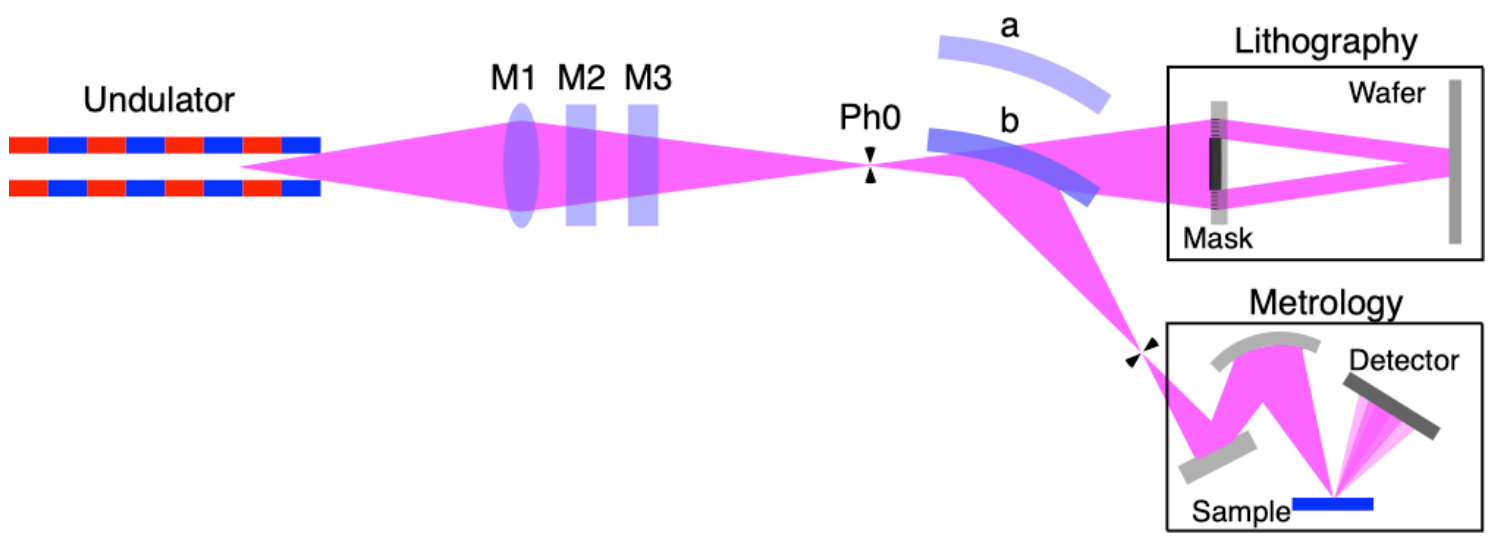

Figure 1. XIL-II Beamline schematics. The XIL-II beamline at the SLS has two branches that can operate alternately. M1 is a toroidal mirror focusing the beam on the $\mathrm{Ph} 0$ plane. M2 is a flat mirror used to fine tune the beam angle and the M3 is a threeelement mirror system employed as a harmonic rejection filter. $\mathrm{Ph} 0$ is a pinhole plate with a range of customizable apertures and serves as a spatial filter. A drop-down grating is used to monochromatize the beam and direct it to the metrology end-station. When the grating is retracted, the beam goes to the lithography end-station.

\section{EUV interference lithography}

EUV interference lithography (EUV-IL) is a powerful method for advanced nanopatterning ${ }^{2-4}$. The interference lithography end-station at the XIL-II beamline consists of a vacuum chamber where a transmission mask is illuminated with the 
spatially coherent EUV beam and it generates an interference pattern. A wafer coated with an EUV-photosensitive resist is installed on the interference plane and exposed. In a two-beam interference setup (Figure 2), the transmission mask is constituted by two gratings with lines parallel to the beam polarization separated by a beam stop. The first diffraction orders of the two gratings interfere on the sample plane forming a sinusoidal intensity pattern with a period that is independent of the wavelength.

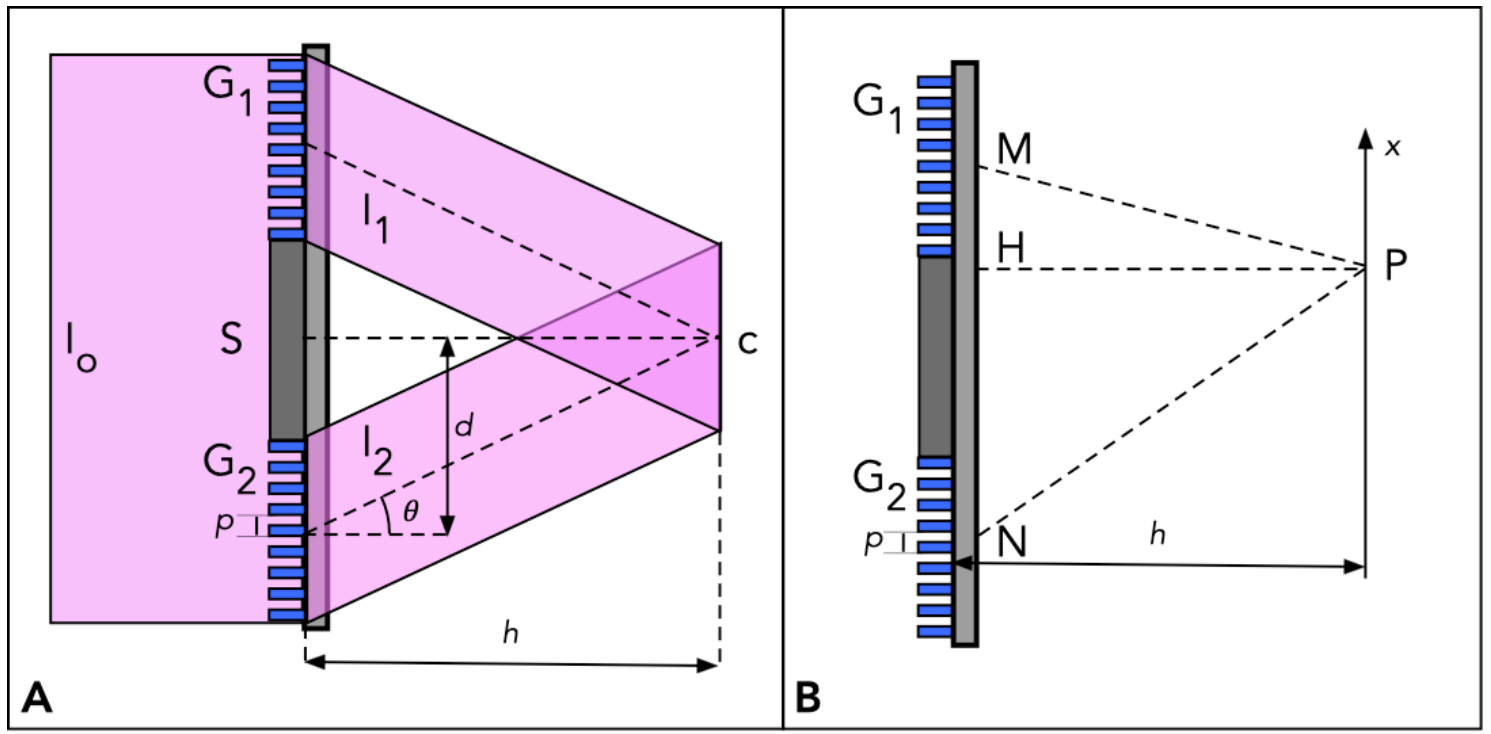

Figure 2. A. Two-beam interference lithography setup. Two hydrogen silsesquioxane (HSQ) gratings $\mathrm{G}_{1}$ and $\mathrm{G}_{2}$ with a pitch $p$ and separated by a distance $2 d$ are printed on a Si3N4 membrane. A nickel beam stop S, blocks the central part of the beam. The first diffraction order beams from the two gratings interfere on the plane $c$ at a distance $h$ from the gratings. B. The optical path difference (OPD) between two rays coming from $\mathrm{G}_{1}$ and $\mathrm{G}_{2}$ is calculated as the difference between NP and MP.

The incoming beam gets diffracted by the two gratings $\mathrm{G}_{1}$ and $\mathrm{G}_{2}$. The distance $d$ between the gratings and their pitch $p$ are chosen so that the +1 -diffraction order beam from $\mathrm{G}_{1}$ and the -1-diffraction order from $\mathrm{G}_{2}$ overlap on the sample plane $c$ at a distance $h$ from the plane of the gratings (Figure 2A). Assuming that the amplitude $E$ of 
the diffracted beams is the same, the interference electric field $E(x)$ on the sample plane can be described as the sum of the electric fields of the two beams:

$$
E_{1}(x)+E_{2}(x)=E \cos \left(k y-\omega t+\phi_{1}(x)\right)+E \cos \left(k y-\omega t+\phi_{2}(x)\right)
$$

where $\phi_{1}(x)$ and $\phi_{2}(x)$ are the phase terms that depend on the interference location along the $x$ axis. Equation 1 can be rearranged as:

$$
E(x)=2 E \cos \left(k y-\omega t+\frac{\phi_{1}(x)+\phi_{2}(x)}{2}\right) \cos \left(\frac{\phi_{1}(x)-\phi_{1}(x)}{2}\right),
$$

and the intensity of the diffraction pattern can be expressed as:

$$
I(x)=E^{2}(x)=2 E^{2} \cos ^{2}\left(\frac{\phi_{1}(x)-\phi_{2}(x)}{2}\right)
$$

The phase difference $\Delta \phi(x)=\phi_{1}(x)-\phi_{2}(x)$ can be calculated from the optical path difference (OPD) of the two interfering beams. The OPD is the difference between the distances PN and PM (Figure 2B):

$$
\mathrm{OPD}=\sqrt{(d+x)^{2}+h^{2}}-\sqrt{(d-x)^{2}+h^{2}},
$$

which for $x \ll d$, can be approximated as:

$$
\mathrm{OPD}=2 x \frac{d}{\sqrt{d^{2}+h^{2}}}
$$

The first-order diffraction angle is given by:

$$
\sin \theta=\frac{\lambda}{p}
$$

therefore, the phase difference is:

$$
\Delta \phi(x)=\operatorname{OPD} \frac{2 \pi}{\lambda}=\frac{4 x \pi}{p} .
$$

Substituting this expression in Equation 3 the aerial image generated by the interference pattern becomes:

$$
I(x)=E^{2}\left(1+\cos \left(\frac{4 \pi x}{p}\right)\right) .
$$

The pattern has a period which is half of the grating pitch. Using the interference from the second-order diffraction would yield a pattern with a period $p / 4 .^{4}$ 
Remarkably, the interference pattern generated with the diffraction gratings is independent of the wavelength which makes it possible to perform EUV interference lithography with non-monochromatic sources and, in the case of the XIL-II undulator source with a $4 \%$ bandwidth at EUV, allows to take advantage of most of the beam energy. This is generally not the case with interference lithography setups using mirrors to split the beam.

The EUV-IL end-station at XIL-II can also be used with more complex diffraction masks to print contact arrays or quasi-periodic structures that found application in several nanofabrication fields ${ }^{5-7}$. This technique provides high-resolution periodic nanostructures over large areas with high throughput. The unique application of the method is providing high-resolution and well-defined aerial images for testing of EUV materials. Owing to the simplicity of the technology that doesn't rely on expensive and complex EUV optical components, it provides higher resolution than the current EUV scanners, enabling timely development of resists for high-NA EUV lithography. Other advantages include the inherently large depth-of-focus and the ability to generate a virtually perfect sinusoidal aerial image as demonstrated by equation 8 . If necessary, it is also possible to vary the contrast of the aerial image by performing an additional exposure using the zero-order light transmitted by one of the two gratings ${ }^{8}$ and thus emulating the flare of a standard projection lens system. In normal operating conditions, the maximum resolution reached is $7 \mathrm{~nm}$ as shown in Figure 3. Using special gratings and hydrogen silsesquioxane (HSQ) resist, it was also possible to print lines with $6 \mathrm{~nm}$ half pitch ${ }^{9}$. 

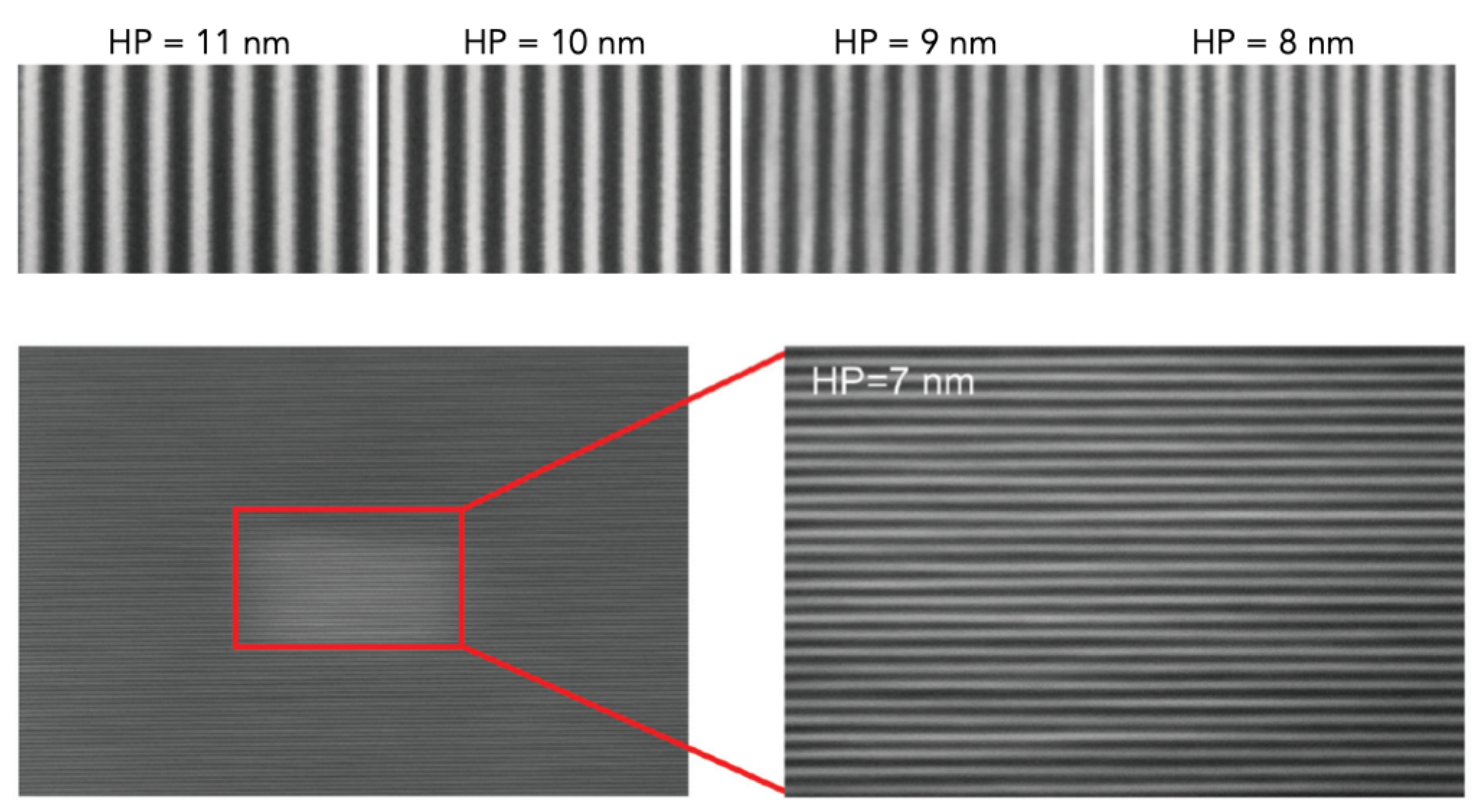

Figure 3. Line-space patterns generated using the XIL-II interference lithography setup with HSQ resist. Image modified from $\left[{ }^{10}\right]$.

\section{Lensless EUV photomask metrology}

Since a single mask is used to print thousands of wafers, it is necessary to verify that it remains defect-free at all times. This is done through an inspection process that identifies the presence of potential defects on the mask, and a review process in which the identified defects are studied in detail, repaired, repair results are verified and finally inspected again to ensure a defect-free mask. The EUV photomask consists of a low thermal expansion substrate of $15 \times 15 \times 6 \mathrm{~mm}$ with a Mo/Si multilayer coating designed to reflect EUV light $(\lambda=13.5 \mathrm{~nm})$ at $6^{\circ}$ incidence and a backside coating of chromium nitride. The multilayer has a Ru capping layer of about $2 \mathrm{~nm}$ and is coated with an absorber layer of TaBN with a thickness of $70 \mathrm{~nm}$. The pattern, defined by etching the absorber layer, has critical dimension (CD) values that can be as low as 60$80 \mathrm{~nm}$ for the current technology node. In a EUV scanner, the mask is illuminated by an incoherent EUV beam with a chief ray angle of $6^{\circ}$ and a complex reflective optical system projects an image of the absorber pattern on a silicon wafer with a 
demagnification factor of 4 . The mask is also protected by a pellicle, transparent to EUV and standing $2.5 \mathrm{~mm}$ above the mask surface to avoid the contamination from fall-on particles.

A critical defect is defined as an alteration of the mask that causes the printed device to malfunction. A broken line or a bridge between two isolated features are examples of such defects. Defects can originate from manufacturing errors in the absorber pattern, from contamination of the mask surface or from imperfections in the mask substrate or in the multilayer deposition that are often referred to as "buried" or "phase" defects because they create a phase disturbance in the reflected beam (Figure 3). Absorber defects with the size of a fraction of the CD and buried defects with a FWHM of $50 \mathrm{~nm}$ and a height of just $0.6 \mathrm{~nm}$ can already have a significant impact in the production yield.

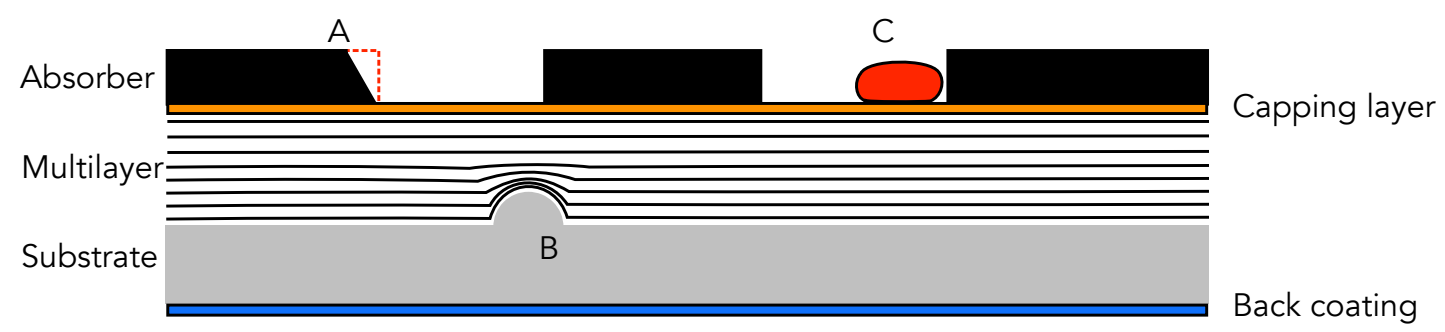

Figure 4. EUV photomask. The structure of the EUV mask consists of a low thermal expansion material substrate with a chromium back side, a Mo/Si multilayer coating capped with a Ru layer and a patterned absorber layer. Mask defects can originate from patterning error (A) from imperfections in the substrate (B) or from the contamination of the mask surface $(\mathrm{C})$.

The goal of the mask inspection process is to identify the potential location of critical defects with an accuracy of $10 \mathrm{~nm}$ or less within a few hours. The current mask inspection technology is based on DUV illumination $(\lambda=193 \mathrm{~nm})$. DUV mask inspection systems can work in die-to-die mode (comparing two areas of the mask with 
the same pattern) and in die-to-database mode (comparing the mask image with an image calculated from the mask design). DUV mask inspection tools are substantially high-NA microscopes and rely on the differential measurement of the mask pattern and a reference for defect detection. Current DUV mask inspection systems can detect defects as small as $60 \times 60 \mathrm{~nm}$. These tools could be used to inspect EUV reticles, however, aside from their resolution limitations, it is important to perform mask inspection at EUV, since defects can have a strong wavelength-dependent response. This is obvious for buried defects, because of the different penetration depth of EUV and DUV into the multilayer, but holds true for absorber defects as well as they can be transparent to EUV and opaque to DUV or vice versa ${ }^{11},{ }^{12}$.

As of today, there is no commercial tool for EUV mask inspection available on the market despite the general consensus of the need for reliable mask metrology solutions to reduce the potential yield loss in the production. In this framework, mask inspection approaches based on lensless imaging are starting to attract more attention, since they represent a way around the costs and the technological challenges posed by the standard imaging techniques.

The RESCAN project provided the first proof of principle for the use of ptychography for EUV mask inspection. RESCAN is a lensless microscope dedicated to EUV mask defect inspection operating at the XIL-II beamline of the Swiss Light Source. RESCAN's working principle consists in collecting images of the sample and comparing them to the image of a reference die or to an aerial image calculated from the mask's design to identify the presence of defects, as shown in Figure 6. The images of the sample are obtained through ptychography, which allows us to retrieve both the phase and the magnitude of the mask surface. RESCAN has been a successful test bed for lensless-based mask inspection and proved to be an ideal tool to develop a 
vibration-robust reconstruction algorithm suitable for use in reflection mode as required by the characteristics of EUV photomasks.

Since RESCAN is based on CDI, the EUV beam needs to be temporally and spatially coherent. For this reason, the beamline monochromator selects a bandwidth $\lambda / \Delta \lambda=1500$. The illumination system of the microscope is outlined in Figure 5. Its simplicity and the absence of optical elements in close proximity to the sample makes it ideal to inspect pellicle-protected EUV masks. This capability is unique to the lensless imaging approach as no EUV inspection or review tool based on standard imaging is currently equipped to operate on samples covered with a pellicle.

In addition to this, the CDI approach used in RESCAN makes it possible to characterize the pellicle-induced flare and monitor the presence of large defects on the pellicle surface as we proved in a recent experiment. ${ }^{13}$

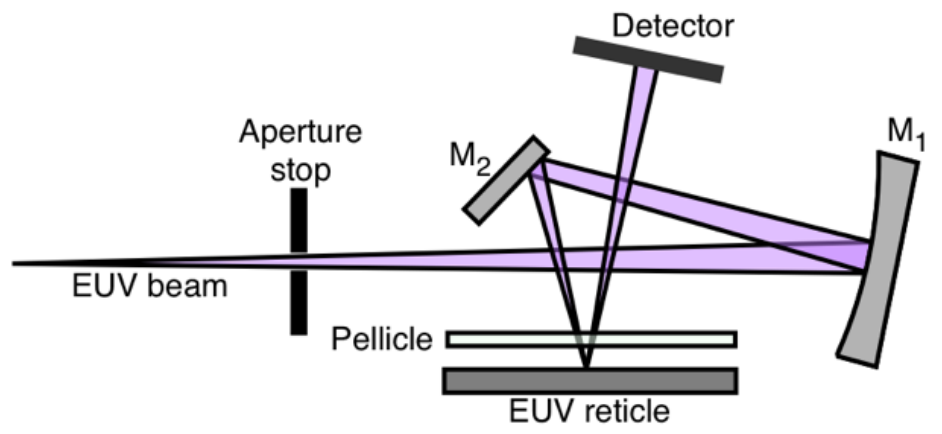

Figure 5. RESCAN optical layout. The size of the EUV beam from the SLS synchrotron is defined by a circular stop with a variable diameter. The beam is focused by a toroidal mirror $\left(\mathrm{M}_{1}\right)$ with a focal length of $120 \mathrm{~mm}$. A flat folding mirror $\left(\mathrm{M}_{2}\right)$ directs the beam on the sample with an angle of incidence of $6^{\circ}$. The beam is focused on the mask surface and, in case a protective pellicle is present, it goes through the membrane twice. The diffraction pattern from the mask is recorded by an EUV CCD. 
Another benefit of CDI is the fact that the image reconstruction includes the phase of the object. This allows a complete characterization of the 3D features on EUV masks. In particular, it allows to detect the presence and the extent of defects buried under the multilayer (See 3B). These defects can be smoothed by the multilayer deposition and be virtually undetectable by SEM or AFM inspection, however because of the penetration of EUV light into the multilayer, they can have an image contrast as high as absorber features.

Defect inspection in RESCAN is performed comparing the reconstructed image with a reference obtained from another area on the mask (die-to-die mode) or calculated from the known mask design (die-to-database mode). To demonstrate the effectiveness of our approach, the defect sensitivity evaluation has been assessed with two specifically designed experiments. ${ }^{14} \mathrm{We}$ fabricated a sample with a test structure with a CD of 200 $\mathrm{nm}$ and absorber defects of different type and size ranging from $200 \mathrm{~nm}$ to $20 \mathrm{~nm}$ (Figure 6A). We calculated the aerial image expected from a defect-free version of the sample (Figure 6B,E) and we compared it to the reconstructed image (Figure 6D). The sample's defect map is shown in Figure 6F where the defects are highlighted in color. The single defect signals are summarized in the array displayed in Figure 7 where it can be seen that the smallest absorber defect detected with RESCAN in die to database mode has a size of $50 \times 50 \mathrm{~nm}^{2}$.

A sample with programmed phase structures was used to evaluate the sensitivity of RESCAN to buried defects. The sample was fabricated depositing $3.5 \mathrm{~nm}$-high carbon discs on a silicon substrate and covering them with a Mo/Si multilayer. An absorber pattern was then deposited on the multilayer and the sample was inspected in RESCAN. The amplitude and phase maps of the reconstructed image are shown in Figure 8 . A bump on a flat mirror would induce a phase shift given by: 


$$
\Delta \phi=\frac{4 \pi h}{\lambda \cos \theta}
$$

where $h$ is the height of the bump, $\lambda$ is the wavelength and $\theta$ is the angle of incidence if the illumination. This formula does not account for the smoothing of the defect induced by the multilayer deposition though and for a more accurate estimate of the phase difference it is necessary to know the profile of the bump across the multilayer thickness and perform an accurate simulation of the light propagation through the sample. ${ }^{15}$ In this case, a simulation based on an accurate model predicted a phase difference $\Delta \phi_{\text {sim }}=0.96 \pi$ for the $3.5 \mathrm{~nm}$ bumps shown in Figure 8 , and the actual value measured by RESCAN was $\Delta \phi=(0.95 \pm 0.02) \pi$.

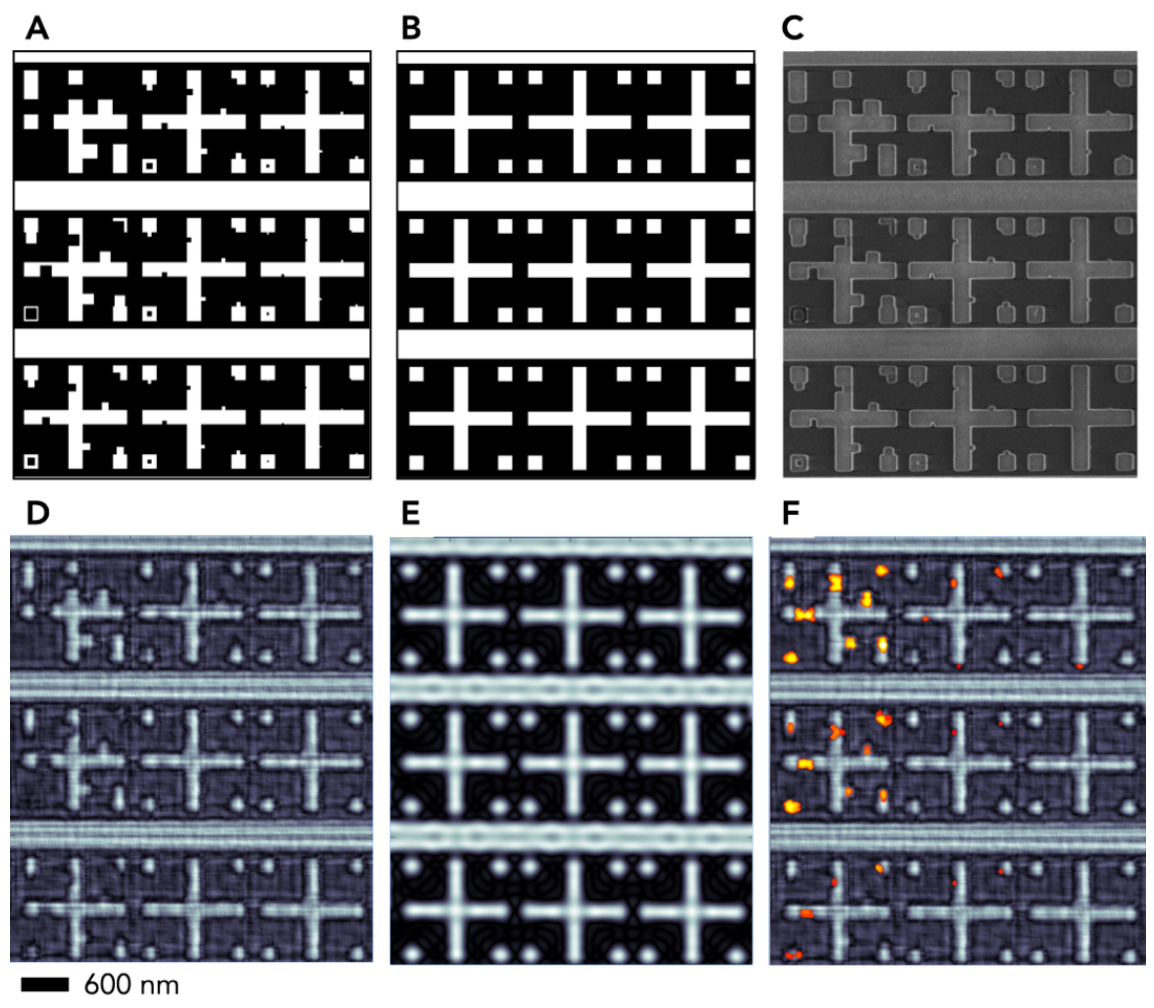

Figure 6. Experimental evaluation of the sensitivity of RESCAN to absorber defects. A. The layout of the sample with programmed absorber defects ranging from $200 \mathrm{~nm}$ (top right) to $20 \mathrm{~nm}$ (bottom left). B. The layout of the sample without the defects. C. An SEM image of the fabricated sample. The size of the structures and the defects was verified with an accuracy of $\pm 1.0 \mathrm{~nm}$. D. Sample image reconstructed with RESCAN. 
E. Sample aerial image calculated from the defect-free design (B). F. Defect map obtained comparing D and E. The defect signal is shown in color in Figure 7.

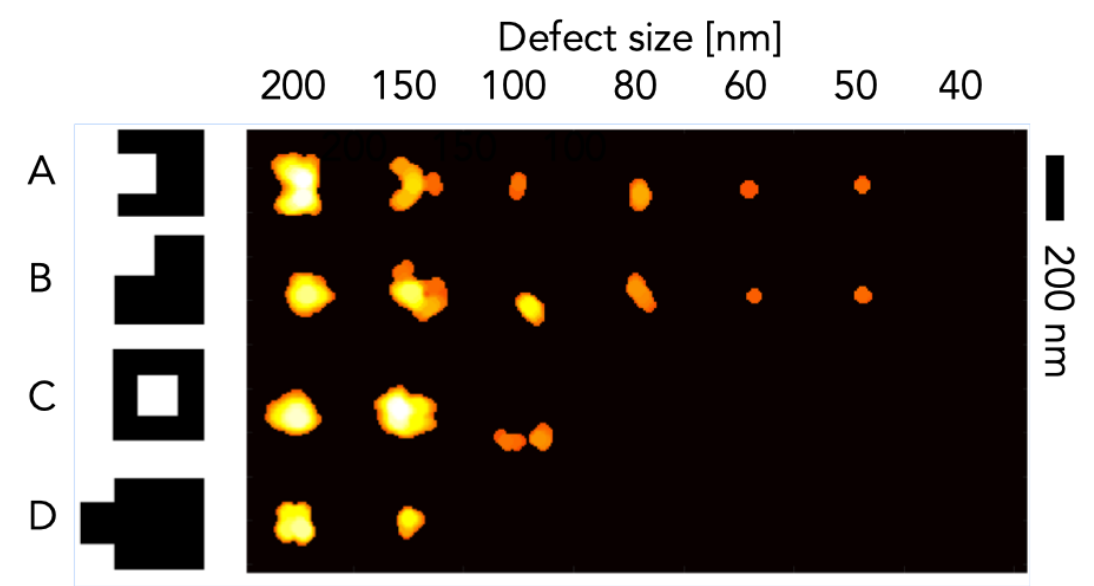

Figure 7. The overview of the measured defect signals on the absorber defect test sample shown in Figure 6. A. Line intrusion. B. Corner intrusion. C. Pin-dot defect. D. Line extrusion. Figure reproduced from $\left[{ }^{14}\right]$.
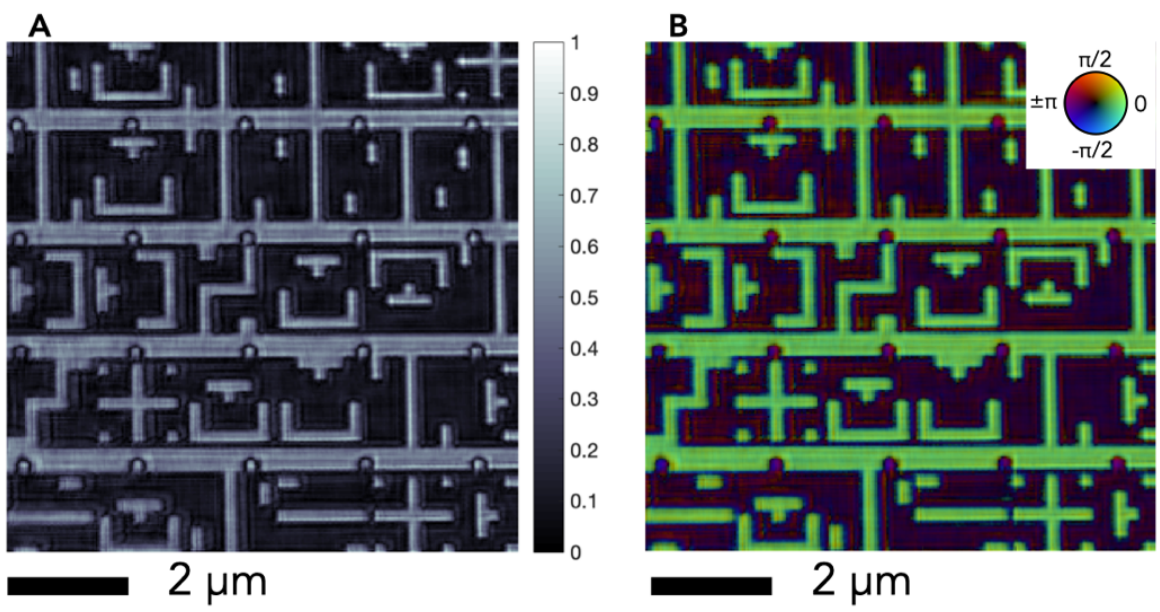

Figure 8. Experimental evaluation of the sensitivity of RESCAN to absorber defects. A. Detail of the reconstructed magnitude map of the phase defect sample. The absorber pattern has a CD of $200 \mathrm{~nm}$ and horizontal trenches of $400 \mathrm{~nm}$. The phase defects are generated by carbon pillars of $200 \mathrm{~nm}$ diameter and $3.5 \mathrm{~nm}$ height and they are aligned with the horizontal tranches. The defects contour is visible in the magnitude map. B. Detail of the reconstructed phase map of the sample. The phase of defects is visualized on a color scale. Figure reproduced from $\left[{ }^{14}\right]$. 


\section{Conclusion}

The XIL-II beamline at the Swiss Light Source is dedicated to EUV research for semiconductor fabrication and it makes use of the coherence properties of the synchrotron beam to study and develop EUV interference and diffraction-based techniques for lithography and metrology. The beamline is equipped with two endstations.

The interference lithography end-station is a nanofabrication facility and a testing platform for EUV resists. The simplicity of the IL technology makes it possible to experiment with different materials and environmental conditions and to test the materials at resolutions beyond the resolution of scanners which makes it an ideal test platform for new EUV resists designed for future technology nodes.

The coherent diffraction imaging end-station is a lensless EUV microscope dedicated to EUV mask inspection. Lensless imaging requires a high temporal coherence which is provided by a monochromator installed on the beamline. RESCAN proved to be a flexible tool that can address multiple EUV lithography-related metrology issues. We demonstrated die-to-die and die-to-database mask inspection with a defect sensitivity of $50 \times 50 \mathrm{~nm} .{ }^{14}$ Thanks to the absence of optical elements in close proximity of the sample, we were able to perform the first through-pellicle mask inspection demonstration. ${ }^{13}$ Finally, we performed a preliminary study of different mask absorber stacks to characterize their performance in terms of image fidelity and contrast. ${ }^{16}$

The RESCAN microscope has been developed as a proof-of-concept platform and we use it to develop a method that can be a valid alternative to standard mask inspection 
techniques. The microscope is synchrotron-based method, but it has the potential to be developed as a commercial, standalone platform if equipped with a suitable source. ${ }^{17}$

\section{References}

1. V. Bakshi, Ed., EUV Lithography, Second Edition, SPIE (2018) [doi:10.1117/3.2305675].

2. M. Wei et al., "Patterning a 50-nm period grating using soft $\mathrm{x}$-ray spatial frequency multiplication,” J. Vac. Sci. Technol. B Microelectron. Nanom. Struct. 12(6), 3648, American Vacuum Society (1994) [doi:10.1116/1.587632].

3. H. H. Solak et al., "Sub-50 nm period patterns with EUV interference lithography," Microelectron. Eng. 67-68, 56-62, Elsevier (2003) [doi:10.1016/S0167-9317(03)00059-5].

4. A. Isoyan et al., "4X reduction extreme ultraviolet interferometric lithography," Opt. Express 16(12), 9106, Optical Society of America (2008) [doi:10.1364/OE.16.009106].

5. D. Kazazis, L.-T. Tseng, and Y. Ekinci, "Improving the resolution and throughput of achromatic Talbot lithography," J. Vac. Sci. Technol. B 36(6), 06J501, American Vacuum Society (2018) [doi:10.1116/1.5048506].

6. X. Wang et al., "Studying resist performance for contact holes printing using EUV interference lithography,” J. Micro/Nanolithography, MEMS, MOEMS 18(01), 1, International Society for Optics and Photonics (2019) [doi:10.1117/1.JMM.18.1.013501].

7. B. Terhalle et al., "Advanced holographic methods in extreme ultraviolet interference lithography," 8 September 2011, 81020V, International Society for Optics and Photonics [doi:10.1117/12.893733].

8. Z. Tasdemir et al., "Contrast matching of line gratings obtained with NXE3XXX and EUV- interference lithography," in International Conference on Extreme Ultraviolet Lithography 2017 10450, P. A. Gargini et al., Eds., p. 65, SPIE 
(2017) [doi:10.1117/12.2280541].

9. D. Fan and Y. Ekinci, "Photolithography reaches $6 \mathrm{~nm}$ half-pitch using extreme ultraviolet light," J. Micro/Nanolithography, MEMS, MOEMS 15(3), 033505, International Society for Optics and Photonics (2016) [doi:10.1117/1.JMM.15.3.033505].

10. N. Mojarad et al., "Single-digit-resolution nanopatterning with extreme ultraviolet light for the $2.5 \mathrm{~nm}$ technology node and beyond," Nanoscale 7(9), 4031-4037, The Royal Society of Chemistry (2015)

[doi:10.1039/C4NR07420C].

11. P. Mangat et al., "Mask blank defect printability comparison using optical and SEM mask and wafer inspection and bright field actinic mask imaging," 9 July 2015, 96580E, International Society for Optics and Photonics [doi:10.1117/12.2201048].

12. I. Mochi et al., "Actinic imaging of native and programmed defects on a fullfield mask," in Proc. of SPIE 7636, B. M. La Fontaine, Ed., p. 76361A, International Society for Optics and Photonics (2010) [doi:10.1117/12.846670].

13. I. Mochi et al., "Experimental evaluation of the impact of carbon nanotube EUV pellicles on reticle imaging," J. Micro/Nanolithography, MEMS, MOEMS 18(01), 1, International Society for Optics and Photonics (2019) [doi:10.1117/1.JMM.18.1.014002].

14. I. Mochi et al., "Amplitude and phase defect inspection on EUV reticles using RESCAN," in Proc. of SPIE, Extreme Ultraviolet (EUV) Lithography X 10957(Cdi), N. M. Felix and K. A. Goldberg, Eds., p. 29, SPIE (2019) [doi:10.1117/12.2515160].

15. M. Upadhyaya et al., "Level-set multilayer growth model for predicting printability of buried native extreme ultraviolet mask defects,” J. Vac. Sci. Technol. B, Nanotechnol. Microelectron. Mater. Process. Meas. Phenom. 33(2), 021602 (2015) [doi:10.1116/1.4913315].

16. S. Fernandez et al., "A comparative study of EUV absorber materials using lensless actinic imaging of EUV photomasks," in Proc.SPIE 10583, pp. 1058310583-10588 (2018) [doi:10.1117/12.2297381]. 
17. Y. Ekinci et al., "A high-brightness accelerator-based EUV source for metrology applications," in Photomask Technology 2018 10810, J. H. Rankin and E. E. Gallagher, Eds., p. 33, SPIE (2018) [doi:10.1117/12.2501930]. 